\title{
SECONDARY EXTINCTION CORRECTION USED IN POLE DENSITY MEASUREMENTS BY X-RAY DIFFRACTION
}

\author{
I. TOMOV and K. IVANOVA* \\ Institute of Physical Chemistry, Bulgarian Academy of Sciences, \\ 1113 Sofia, Bulgaria \\ *Central Laboratory of Solar Energy and New Energy Sources, 1184 Sofia, \\ Bulgaria
}

(Received 10 November 1995)

\begin{abstract}
A new X-ray diffraction method for a more accurate determination of film pole densities is developed by introducing a secondary extinction correction. To this end an equation based on the kinematic theory of X-ray diffraction and the mosaic crystal model is derived. Pole densities were computed either by using the two order reflections techniques or by the least-square method. The discussion of results shows that the correct study of film structure by X-ray diffraction requires in general the secondary extinction to be taken into account.
\end{abstract}

KEY WORDS: Secondary extinction, pole density, films, X-ray diffraction.

\section{INTRODUCTION}

In a previous paper it was established that the integrated intensity of the strongest $\mathrm{X}$ ray diffraction lines of textured films was lower than the kinematic theory premises (Tomov 1976). Since the electrodeposited metal films are of fine crystalline lamellar structure this effect was ascribed to the secondary extinction. The secondary extinction decreased in a different extent the integrated intensity of each of the diffraction lines. The intensity decrease would cause a systematical error in pole density measurements which was different in each of the measured directions and, in addition, it was intolerably high in the main texture component direction. If it was accounted for the influence of the secondary extinction on the observed intensity, the systematical error would be canceled and the accuracy in the pole density measurements would markedly be raised. It is the purpose of the present paper to give a derivation of an improved method for more accurate pole density measurements in textured "infinitely" thick films. Because of the natural macrofeatures of films (i.e., large lateral dimensions) the considerations to be made will use the results concerning the secondary extinction correction for the symmetrical Bragg case reflection (Chandrasekhar 1960, Zachariasen 1963).

In memory of Prof. Dr. h.c. William Hsun Hu. 


\section{INTRODUCING THE SECONDARY EXTINCTION CORRECTION IN THE INTEGRATED INTENSITY OF A TEXTURED FILM}

The reflecting power of a real crystal is in between that of an ideally imperfect crystal and a perfect one (James 1960). The mosaic structure model of a real crystal was proposed by Darwin, 1922, to account for its reflecting power which is affected by extinctions. The model represents a real crystal as consisting of slightly misoriented, $\mathrm{X}$-ray optically independent blocks distributed in depth randomly.

Extinctions in a mosaic crystal structure are the power losses caused by the production of a diffracted beam. If the mosaic structure consists of large crystal blocks, the extinction within each ideal block is called primary extinction. When it is a fine crystalline mosaic structure, the power loss due to diffraction in blocks that are traversed by the incident beam before it reaches the particular block under consideration is called secondary extinction.

The theoretical study of secondary extinction by Darwin, 1922, Chandrasekhar, 1960 and Zachariasen, 1963, 1965, 1967, has been confined to crystals in which the domain distribution function is much wider than the diffraction pattern of a single domain. According to the theory secondary extinction is an inherent property of slightly misoriented fine crystalline structures with blocks distributed in depth randomly. In fact, such a distribution is approximately similar to that of sharp growth textures. The last is a premise to apply the considerations of the secondary extinction theory to studies of film texture. The motivation to introduce a secondary extinction correction in observed intensity is related to the accuracy in pole density measurements.

In the symmetrical Bragg case reflection by a parallel plate as a first-order correction for the secondary extinction Chandrasekhar, 1960 and Zachariasen, 1963 showed that one should use an effective absorption coefficient

$$
\mu_{\varepsilon}=\mu+2 \mathrm{gQ} \frac{\mathrm{p}_{2}}{\mathrm{p}_{1}^{2}}
$$

where $\mu$ is a linear absorption coefficient, $g$ is a secondary extinction coefficient which is a constant for the crystal depending on the nature of the mosaic, $Q$ is the reflectivity of a lattice plane (hkl). For the case of a monochromator with Bragg angle $\theta_{0}$

and

$$
\mathrm{p}_{1}=\frac{1+\cos ^{2} 2 \theta_{0} \cos ^{2} 2 \theta}{1+\cos ^{2} 2 \theta_{0}}
$$

$$
\mathrm{p}_{2}=\frac{1+\cos ^{2} 2 \theta_{0} \cos ^{4} 2 \theta}{1+\cos ^{2} 2 \theta_{0}}
$$

where $\theta$ is the Bragg angle of the diffraction line.

\section{"Infinitely" Thick Textured Film}

The following considerations are based on the supposition that the diffraction phenomena in films are similar to those in mosaic crystals. To describe the secondary extinction problem the film will be considered first, as an imaginary "ideally imperfect" crystal and second, as a crystal with "mosaic structure" which approximates the real film structure. These terms are related to film reflecting power in two different approxi- 
mations. In principle, diffraction by an "ideally imperfect" film must be obedient to the requirements of the kinematical theory. That means that the diffracted intensity from a film is not affected by extinctions. The integrated intensity $I_{\text {kin }}$ is then expressed by the equation

$$
\frac{\mathrm{I}_{\text {kin }}}{\mathrm{I}_{0} \mathrm{~S}}=\frac{\mathrm{Q}}{2 \mu}
$$

where $I_{0}$ is the intensity of the incident beam, $S$ being its cross section.

Secondly, we suppose that the film is of a mosaic fine crystalline structure. That means that the diffracted intensity is extinguished by secondary extinction only. Then the integrated intensity $I_{m}$ of each of the diffraction lines has to be expressed by the equation

$$
\frac{\mathrm{I}_{\mathrm{m}}}{\mathrm{I}_{0} \mathrm{~S}}=\frac{\mathrm{Q}}{2 \mu_{\varepsilon}}
$$

where $\mu_{\varepsilon}$ is the effective absorption coefficient given by Eq. (1). Dividing eq. (5) by eq. (4) it follows that

$$
\mathrm{I}_{\mathrm{m}}=\frac{\mu}{\mu_{\varepsilon}} \mathrm{I}_{\mathrm{kin}}=\mathrm{yI}_{\mathrm{kin}}
$$

which relates the intensities of the respective diffraction lines of both the imaginary "ideally imperfect" film and the real mosaic fine crystalline structure film. Here $\mathbf{y}$ is a secondary extinction factor which is always smaller than unity. In general, the secondary extinction factor depends on the sample defect structure, the lattice plane reflectivity $\mathbf{Q}$, the Bragg angle $\theta$, the sample thickness $\mathbf{t}$, as well as on the diffracted radiation wave length $\lambda$ (James 1960).

Replacing $\mu_{\varepsilon}$ by its corresponding expression from Eq. (1) transforms Eq. (6) into

$$
\mathrm{I}_{\mathrm{m}}=\frac{\mu}{\mu+2 \mathrm{gQ} \frac{\mathrm{p}_{2}}{\mathrm{p}_{1}^{2}}} \mathrm{I}_{\mathrm{kin}}
$$

Intensity measurements are usually made on relative rather than absolute scale. According to James, 1960 and Zachariasen 1963, 1965, the secondary extinction $\varepsilon$ is proportional to the integrated intensity $I_{k i n}$ of the respective line so that the effective absorption coefficient is given by

$$
\mu_{\varepsilon}=\mu+\varepsilon=\mu+2 \mathrm{kI}_{\mathrm{kin}} \frac{\mathrm{p}_{2}}{\mathrm{p}_{1}^{2}}
$$

where $\mathrm{k}$ is a scale factor depending on film texture sharpness. With Eq. 8 introduced into Eq. 6 the integrated intensity $I_{m}$ of a mosaic film is expressed by

$$
\mathrm{I}_{\mathrm{m}}=\frac{\mu}{\mu+2 \mathrm{kI}_{\mathrm{kin}} \frac{\mathrm{p}_{2}}{\mathrm{p}_{1}^{2}}} \mathrm{I}_{\text {kin }}
$$

Expressing the film kinematical intensity using two order reflections

Eq. (9) has two unknown quantities so that it can be solved using the two order reflection techniques. For some cases of two order reflections the crystallites contributing to each 
of the reflections may be the same as if they belong to one $<\mathrm{hkl}>$ direction. Than Eq. (9) can be rewritten for these reflections as

$$
I_{m}^{\prime}=\frac{\mu}{\mu+2 \mathrm{kI}_{\text {kin }}^{\prime}\left(\frac{\mathrm{p}_{2}}{\mathrm{p}_{1}^{2}}\right)^{\prime}}{I^{\prime}}^{\prime}{ }_{\text {in }}
$$

at the lower Bragg angle $\theta$, and as

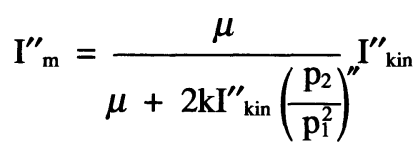

at the higher Bragg angle $\theta^{\prime \prime}$. Since the pole densities of such reflections are equal, the following ratio is valid

$$
\frac{I_{\text {kin }}^{\prime}}{I^{\prime \prime}{ }_{\text {in }}}=\frac{I_{r}^{\prime}}{I_{r}^{\prime \prime}}
$$

where $I_{r}^{\prime}$ and $I^{\prime \prime}$ are the integrated intensities of the respective diffraction lines of a powder standard of the same substance. Dividing Eq. (10) by Eq. (11) and taking into account Eq. (12) gives the following expressions for $\mathbf{k}, I_{\text {kin }}^{\prime}$ and $I^{\prime \prime}$ kin

$$
\begin{aligned}
& k=\frac{\mu\left(I_{m}^{\prime \prime} \frac{I_{r}^{\prime}}{I_{r}^{\prime \prime}}-I_{m}^{\prime}\right)}{2\left\{\frac{I_{r}^{\prime}}{I_{r}^{\prime \prime}}\left[I_{m}^{\prime}\left(\frac{p_{2}}{p_{1}^{2}}\right)^{\prime}-I_{m}^{\prime \prime}\left(\frac{p_{2}}{p_{1}^{2}}\right)^{\prime \prime}+I_{m}^{\prime \prime}\right]-I_{m}^{\prime}\right\}} \\
& I_{k i n}^{\prime}=\frac{\mu\left(I_{m}^{\prime \prime} \frac{I_{r}^{\prime}}{I_{r}^{\prime \prime}}-I_{m}^{\prime}\right)}{2 k\left[I_{m}^{\prime}\left(\frac{p_{2}}{p_{1}^{2}}\right)^{\prime}-I_{m}^{\prime \prime}\left(\frac{p_{2}}{p_{1}^{2}}\right)^{\prime \prime}\right]} \\
& I^{\prime \prime}{ }_{k i n}=\frac{\mu\left(I_{m}^{\prime \prime} \frac{I_{r}^{\prime}}{I_{r}^{\prime \prime}}-I_{m}^{\prime}\right)}{2 k \frac{I_{r}^{\prime}}{I_{r}^{2}}\left[I_{m}^{\prime}\left(\frac{p_{2}}{p_{1}^{2}}\right)^{\prime}-I_{m}^{\prime \prime}\left(\frac{p_{2}}{p_{1}^{2}}\right)^{\prime \prime}\right]}
\end{aligned}
$$

Knowing $\mathbf{k}$, an expression for the intensity $I_{k i n}$ follows from Eq. (9) for any of the measured directions:

$$
\mathrm{I}_{\mathrm{kin}}=\frac{\mu}{\mu-2 \mathrm{kI}_{\mathrm{m}} \frac{\mathrm{p}_{2}}{\mathrm{p}_{1}^{2}}} \mathrm{I}_{\mathrm{m}}
$$

It is possible to make a calculation of kinematical intensities by the least-square method. If $I_{k i n}$ is defined by Eq. (9) the following minimizing condition can be postulated for fitting its mean value by a free varying parameter $\mathrm{k}$, which is an adjustable scale factor (constant for the film):

$$
\sum_{\mathrm{i}}\left[\mathrm{I}_{\mathrm{m}}^{\mathrm{i}}-\mathrm{y}^{\mathrm{i}} \mathrm{I}_{\mathrm{kin}}^{\mathrm{i}}\right]^{2}=\min
$$


Here $\mathbf{i}$ is the number of the measured lines. The expression

$$
P=I_{\text {kin }} / I_{r}
$$

defines the pole density by the integrated intensities of the respective lines of both a kinematically diffracting thick film and a powder standard of the same substance. Eq. (18) can be used for a more accurate pole density determination of infinitely thick films.

\section{SPECIMEN PREPARATION AND EXPERIMENTAL}

The models of our study were electrodeposited nickel films with $<100\rangle+<221>$ texture and a thickness of about $50 \mu \mathrm{m}$. The deposition was carried out on disk copper substrates with a diameter of $30 \mathrm{~mm}$. The $<221>$ - twin component is regularly arisen by firstorder twinning on crystal blocks belonging to the main $\langle 100\rangle$ - texture component (Tomov 1976). In X-ray diffraction sense, the $50 \mu \mathrm{m}$ thick films behaved as bulk metal, since their thickness was greater than the effective depth of X-ray penetration (Tomov 1986). The conditions of electrodeposition were selected in such a way, so as to obtain films with different texture sharpness. To this end we prepare matt and bright nickel films.

The diffraction line measurements of the films were performed by an X-ray diffractometer Philips with $\mathrm{CuK}_{\alpha}$-radiation selected by a graphite focusing monochromator. In relation to the purposes of the investigation, series of diffraction lines of each of the samples were measured, i.e. 111, 200, 220, 311, 222 and 400 ones.

In order to reveal the mosaic structure of nickel films with $<100>+<221>$ texture their microstructure was investigated. The microstructure pictures of both the grown side surface and the cross section were obtained in an electron microscope by Pt-C replicas. The Pt-C replicas were produced after electrolytic polishing and electrolytic etching of the respective surfaces. Polishing was carried out in a $60 \% \mathrm{H}_{2} \mathrm{SO}_{4}$ aqueous solution at about $35^{\circ} \mathrm{C}$ and $40 \mathrm{~A}^{-\mathrm{dm}^{-2}}$ for $20 \mathrm{~s}$. Polished surfaces were etched in a $20 \% \mathrm{HCl}$ methyl alcohol solution at about $35^{\circ} \mathrm{C}$ and current density $0.05 \mathrm{~A} . \mathrm{dm}^{-2}$ for $10 \mathrm{~s}$.

\section{RESULTS AND DISCUSSION}

First, the compatibility of the model used to solve the secondary extinction problem by Darwin, 1922 and Zachariasen, 1963 and the structure of electrodeposited films will be considered. The compatibility of the mosaic structure model with the structure of textured films is illustrated by pictures obtained in an electron microscope using Pt$\mathrm{C}$ replicas. The pictures are indicative for the lamellar structure of nickel films with sharp $<100>+<221>$ texture. Figure 1 reveals the surface morphology (growth surface side) of nickel films whose crystal grains consist of blocks which are separated by twin boundaries. The next pictures (Figure 2) show nickel films cross sections which reveal their columnar structure. The columns consist of blocks which are also separated by twin boundaries. Because of twinning each of the blocks has different orientation 

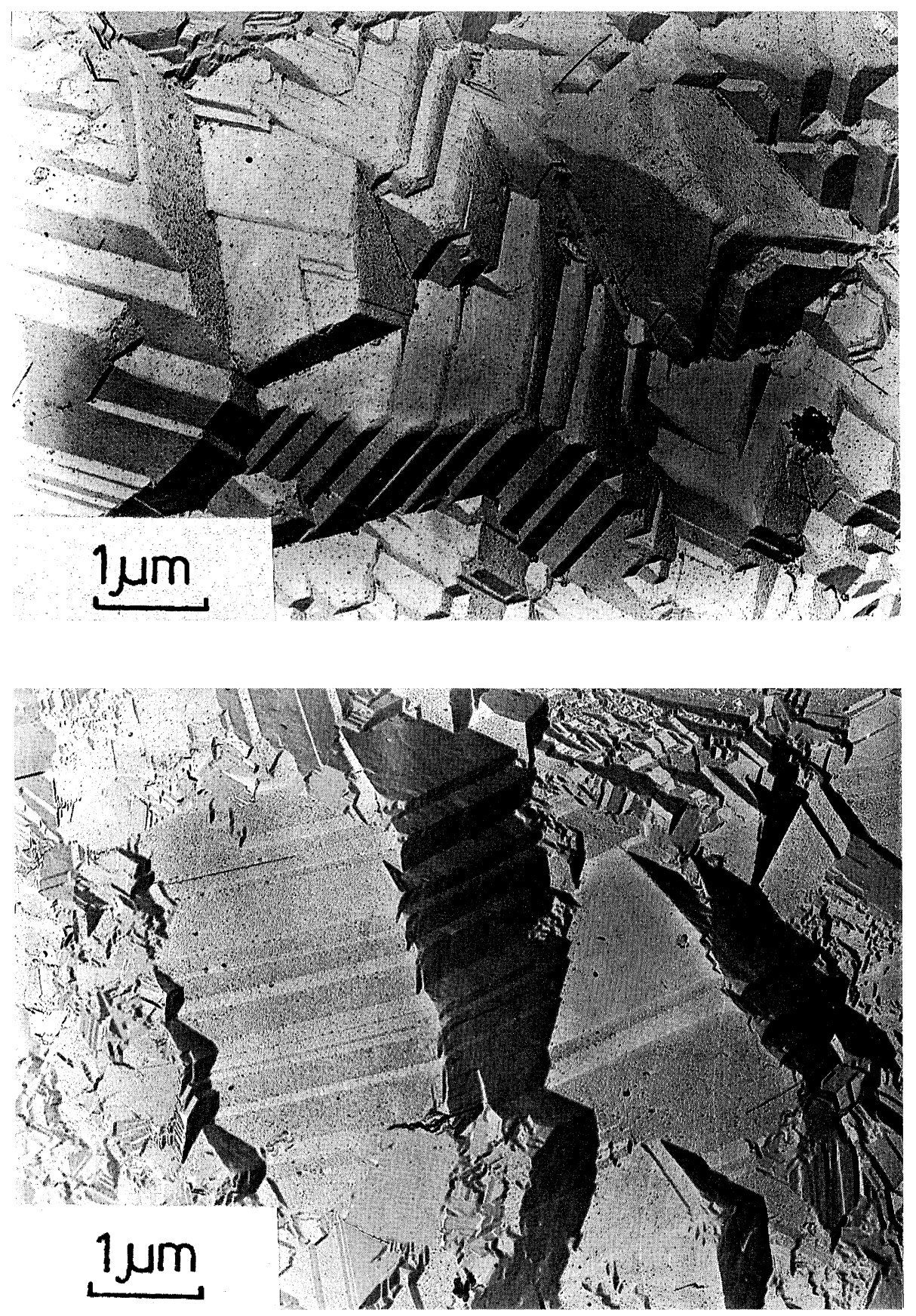

Figure 1 Electron microscope pictures of $\mathrm{Pt}-\mathrm{C}$ replicas showing the surface morphology (grown side surface) of etched nickel films with $\langle 100\rangle+\langle 221\rangle$ texture. In both cases the crystal grains consist of blocks which are separated by twin boundaries. 

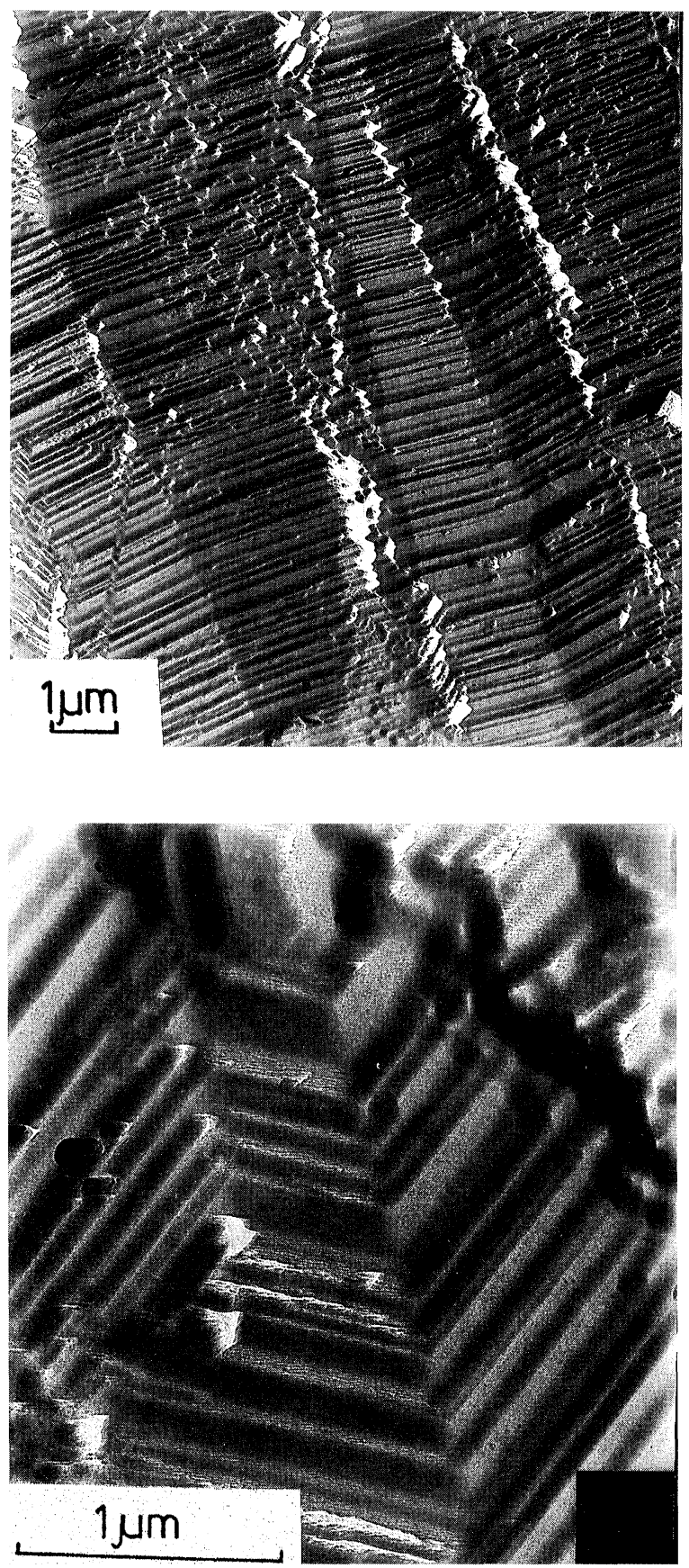

Figure 2 Electron microscope pictures of Pt-C replicas showing the columnar structure of etched cross section of nickel films with $<100\rangle+<221>$ texture. The columns consists of polysynthetic twinned blocks: a - bright nickel cross section; $\mathrm{b}$ - matt nickel cross section. 
with respect to its neighbor implying that they are X-ray optically independent. Moreover, because of multiple twinning during film growth and the accidental character of the twin process, the distance between blocks with the same orientations could not be constant. The last premises random distribution of blocks with equal orientations in the depth of the film. Consequently the cross sections show that crystalline distribution in the film is in some degree similar to that in the mosaic structure model where a requirement is postulated for X-ray optical independence and random distribution of blocks in the depth of the crystal. The cross sections presented are the best visible arguments for compatibility of the mosaic structure model with a real film structure. Taking into account these preliminary results, we could better understand the main results concerning secondary extinction and pole density.

The values of the secondary extinction $\varepsilon$ are shown in Figure 3ab together with the respective X-ray diffraction patterns of two films. Figure $3 a$ represents most of the diffraction lines of nickel film of relatively sharp $<100\rangle+<221>$ texture. The $\varepsilon$-values are arranged below the hkl-indexes of the respective diffraction lines. The ordinary linear absorption coefficient $\mu$ of nickel is given over them. The $\varepsilon$-values are the greatest in the direction of the main texture component $\langle 100\rangle$. In comparison with the $\mu$-coefficient of nickel they amount to about $35 \%$ for the 200 diffraction line and to about $4 \%$ for the 400 diffraction line, respectively. Figure $3 \mathrm{~b}$ shows the $X$-ray diffraction pattern of very sharp $<100>+<221>$ texture. The respective $\varepsilon$-values amount to about $115 \%$ for the 200 diffraction line and to $15 \%$ for the 400 diffraction line compared to the $\mu$-value of nickel. The $\varepsilon$-values are rather smaller in other measured directions presented by the two X-ray diffraction patterns (Figure 3ab). The generalization of these results premises that textured film could be considered as a multicomponent material consisting of several "imaginary substances" of different linear absorption coefficient appropriate to each of the diffraction lines. These diffraction lines are not applicable to any correct estimation of film structure if the secondary extinction correction is not introduced in the observed intensity.

The influence of secondary extinction on the accuracy in pole density measurements is shown in Table 1 . The values of $\varepsilon, \mathrm{P}$ and $\mathrm{P}_{\mathrm{m}}$ quantities are listed in it. The quantity $P_{m}$ is defined by the equation

The difference

$$
P_{m}=I_{m} / I_{r}
$$

$$
\mathbf{P}-\mathbf{P}_{\mathrm{m}}=\Delta \mathbf{P}
$$

is used as a measure of the systematical error caused by the secondary extinction in pole density measurements. In general, the systematical error has the greatest values in the direction of the main texture component. In nearly all the cases the error $\Delta \mathbf{P}_{200}$ is intolerably high. In some of the cases $\Delta \mathbf{P}_{400}$ is considerable and yet many times smaller than $\Delta \mathrm{P}_{200}$. (But in practice one uses preferably 200 pole figure instead of 400 pole figures). The systematical error $\Delta \mathrm{P}_{111}$ exceeds the usual statistical error attainable in $\mathrm{X}$-ray diffraction measurements. For all other directions the influence of secondary extinction on the accuracy in pole density determination is negligibly small. However, these directions are related to very weak diffraction lines.

The $\varepsilon, P$ and $P_{m}$-values are also calculated by the least-square method (Table 2). This method is preferable when the texture represents neither $<100\rangle$ nor $<111\rangle-$ main component giving diffraction line pairs each of them having equal pole density, respectively. The coincidence of the results obtained by the two computing approaches is quite good. 


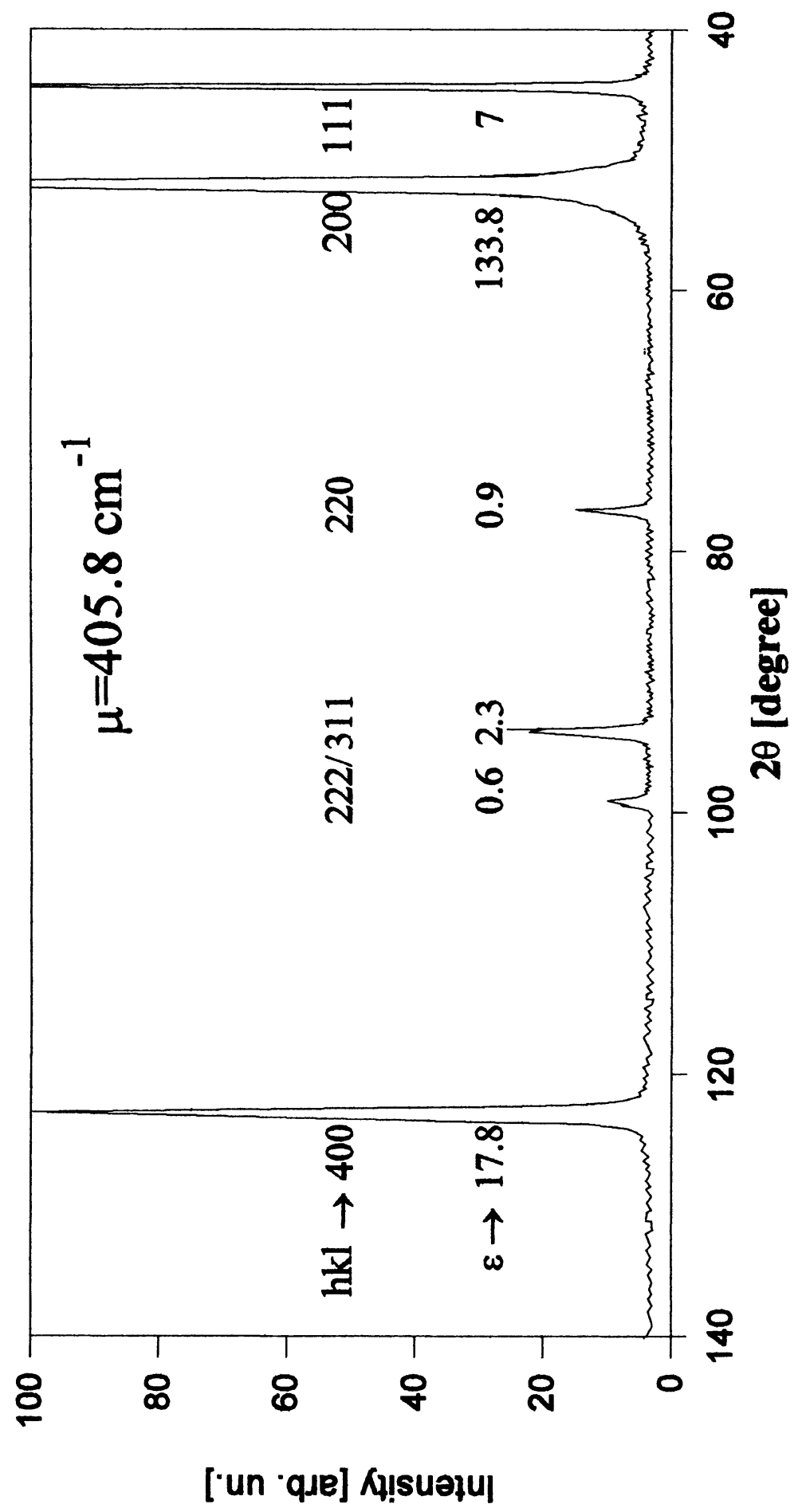




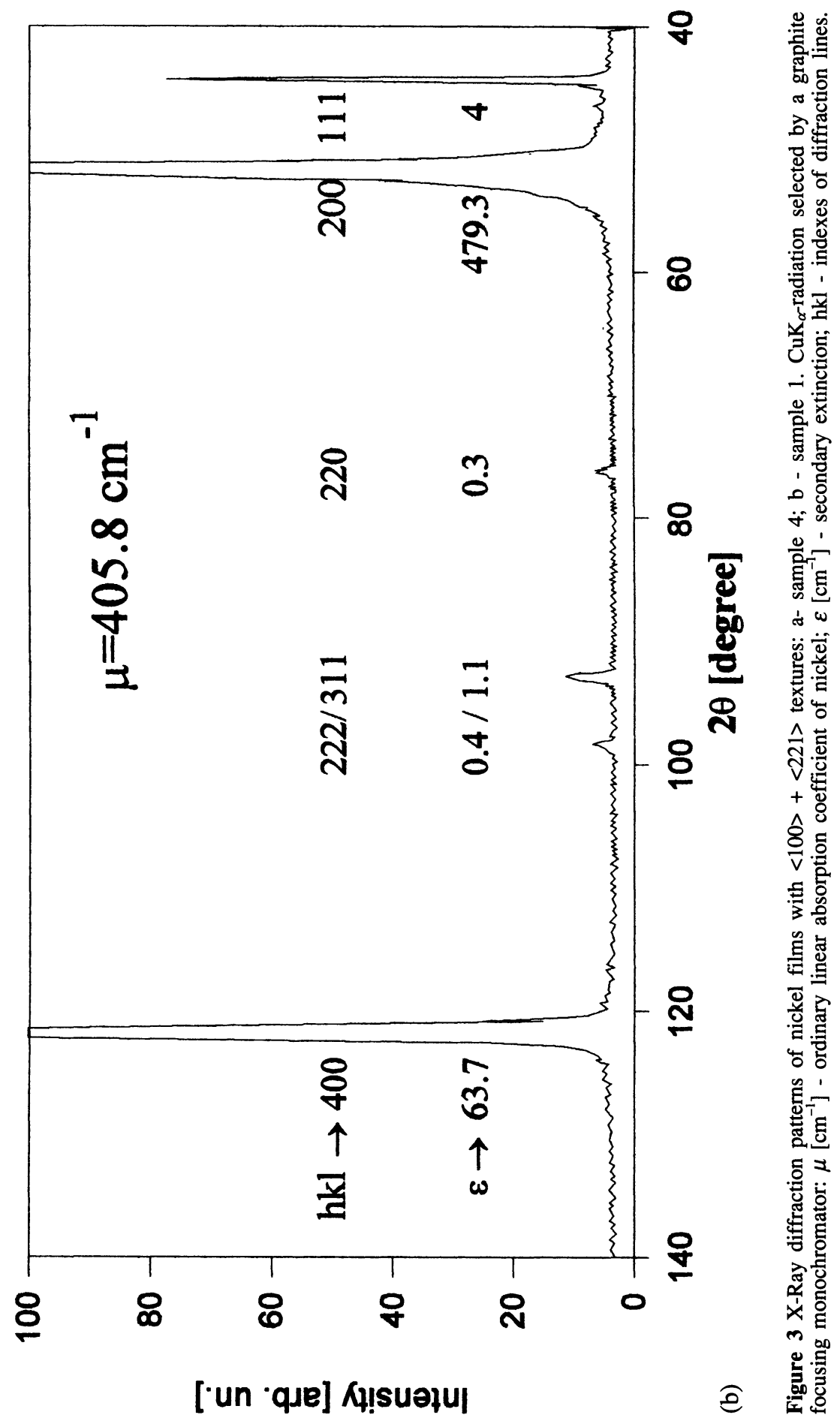


Table 1 Secondary extinction $\varepsilon\left[\mathrm{cm}^{-1}\right]$ and pole densities $\mathrm{P}$ and $\mathrm{P}_{\mathrm{m}}$ calculated by using the simple solution for 200-400 diffraction pair from kinematic integrated intensity $I_{k i n}$ and measured intensity $I_{m}$ of thick nickel film diffraction lines, respectively. Linear absorption coefficient $\mu=405.82 \mathrm{~cm}^{-1}$, $\mathrm{CuK}_{\alpha}$ - radiation and graphite focusing monochromator.

\begin{tabular}{|c|c|c|c|c|c|c|c|}
\hline \multirow[t]{2}{*}{$N$} & \multirow[t]{2}{*}{ Quantity } & \multicolumn{6}{|c|}{$h k l$} \\
\hline & & 200 & 400 & 111 & 222 & 220 & 311 \\
\hline \multirow{3}{*}{1} & $\varepsilon$ & 479.25 & 63.72 & 3.99 & 0.45 & 0.29 & 1.14 \\
\hline & $\mathbf{P}$ & 52.74 & 52.74 & 0.18 & 0.23 & 0.06 & 0.20 \\
\hline & $P_{m}$ & 24.18 & 45.58 & 0.18 & 0.23 & 0.06 & 0.20 \\
\hline \multirow{3}{*}{2} & $\varepsilon$ & 415.17 & 55.20 & 21.26 & 1.76 & 0.59 & 4.72 \\
\hline & $\mathrm{P}$ & 35.25 & 35.25 & 0.73 & 0.69 & 0.09 & 0.63 \\
\hline & $P_{m}$ & 17.42 & 31.03 & 0.70 & 0.69 & 0.09 & 0.62 \\
\hline \multirow{3}{*}{3} & $\boldsymbol{\varepsilon}$ & 201.09 & 26.74 & 16.63 & 1.34 & 1.40 & 4.45 \\
\hline & $\mathbf{P}$ & 20.78 & 20.78 & 0.70 & 0.64 & 0.27 & 0.72 \\
\hline & $P_{m}$ & 13.89 & 19.49 & 0.67 & 0.64 & 0.27 & 0.71 \\
\hline \multirow{3}{*}{4} & $\boldsymbol{\varepsilon}$ & 133.78 & 17.79 & 7.01 & 0.63 & 0.94 & 2.32 \\
\hline & $\mathbf{P}$ & 20.08 & 20.08 & 0.43 & 0.44 & 0.26 & 0.55 \\
\hline & $P_{m}$ & 15.10 & 19.24 & 0.42 & 0.44 & 0.26 & 0.54 \\
\hline \multirow{3}{*}{5} & $\boldsymbol{\varepsilon}$ & 11.71 & 1.56 & 2.69 & 0.23 & 0.02 & 1.18 \\
\hline & $\mathrm{P}$ & 9.32 & 9.32 & 0.87 & 0.83 & 0.03 & 1.48 \\
\hline & $P_{m}$ & 9.06 & 9.29 & 0.86 & 0.83 & 0.03 & 1.47 \\
\hline
\end{tabular}

Table 2 Secondary extinction $\varepsilon\left[\mathrm{cm}^{-1}\right]$ and pole densities $\mathrm{P}$ and $\mathrm{P}_{\mathrm{m}}$ calculated by the least-square method using the 111-222 diffraction pair and the 200-400 diffraction pair. Linear absorption coefficient $\mu=405.82 \mathrm{~cm}^{-1}, \mathrm{CuK}_{\alpha}$ - radiation and graphite focusing monochromator.

\begin{tabular}{|c|c|c|c|c|c|c|c|}
\hline \multirow[t]{2}{*}{$N$} & \multirow{2}{*}{ Quantity } & \multicolumn{6}{|c|}{$h k l$} \\
\hline & & 200 & 400 & 111 & 222 & 220 & 311 \\
\hline \multirow{3}{*}{1} & $\varepsilon$ & 484.15 & 64.37 & 4.56 & 0.40 & 0.29 & 1.15 \\
\hline & $\mathbf{P}$ & 52.89 & 52.89 & 0.20 & 0.20 & 0.06 & 0.20 \\
\hline & $P_{m}$ & 24.18 & 45.58 & 0.18 & 0.23 & 0.06 & 0.20 \\
\hline \multirow{3}{*}{2} & $\boldsymbol{\varepsilon}$ & 410.46 & 54.57 & 20.46 & 1.79 & 0.59 & 4.68 \\
\hline & $\mathbf{P}$ & 35.14 & 35.14 & 0.71 & 0.71 & 0.09 & 0.63 \\
\hline & $P_{m}$ & 17.42 & 31.03 & 0.70 & 0.69 & 0.09 & 0.62 \\
\hline \multirow{3}{*}{3} & $\boldsymbol{\varepsilon}$ & 194.70 & 25.89 & 15.48 & 1.36 & 1.36 & 4.33 \\
\hline & $\mathbf{P}$ & 20.66 & 20.66 & 0.67 & 0.67 & 0.27 & 0.72 \\
\hline & $P_{m}$ & 13.89 & 19.49 & 0.67 & 0.64 & 0.27 & 0.71 \\
\hline \multirow{3}{*}{4} & $\varepsilon$ & 134.65 & 17.90 & 7.13 & 0.62 & 0.95 & 2.33 \\
\hline & $\mathbf{P}$ & 20.10 & 20.10 & 0.43 & 0.43 & 0.26 & 0.55 \\
\hline & $P_{m}$ & 15.10 & 19.24 & 0.42 & 0.44 & 0.26 & 0.54 \\
\hline \multirow{3}{*}{5} & $\varepsilon$ & 6.90 & 0.92 & 1.56 & 0.14 & 0.01 & 0.70 \\
\hline & $\mathbf{P}$ & 9.26 & 9.26 & 0.85 & 0.85 & 0.03 & 1.47 \\
\hline & $\mathbf{P}_{\mathrm{m}}$ & 9.06 & 9.29 & 0.86 & 0.83 & 0.03 & 1.47 \\
\hline
\end{tabular}




\section{CONCLUSION}

A new X-ray diffraction method for pole density measurement of textured films has been described. The method accounts for the influence of secondary extinction on the observed intensity. A simple solution for the unknown quantities is given, using the two order reflections technique (i.e., using the 200 and the 400 reflection pair due to the contribution of the main $<100>$-texture component). Calculation of unknown quantities could also be made by the least-square method. The latter is independent of the texture type, since it does not require availability of the reflection pair of the main texture component as the former method does. Application of the secondary extinction correction to the textured films is found to be extremely useful in determining the correct pole densities. It may be anticipated that the high precision in any X-ray diffraction characterization of films could be further attained using this correction which tends to become very high in the case of rather sharp textures.

\section{Acknowledgments}

This work was supported by the Bulgarian National Fund for Scientific Research under Contract No. F-455/1994.

\section{References}

Chandrasekhar, S. (1960). Advances in Physics, 9, 363.

Darwin, C. C. (1922). Phil. Mag., 43, 800.

James, R. W. (1960). The Optical Principles of the Diffraction of X-Rays. London. Bell.

Tomov, I. (1976). Izv. Chem., 9, 192.

Tomov, I. (1986). pss(a), 95, 397.

Zachariasen, W. H. (1963). Acta Cryst., 16, 1139.

Zachariasen, W. H. (1965). Trans. Amer. Crystal. Association, 1, 33.

Zachariasen, W. H. (1967). Acta Cryst., 23, 558. 